\title{
Impact of Protein Intake during Weight Loss on Preservation of Fat-Free Mass, Resting Energy Expenditure, and Physical Function in Overweight Postmenopausal Women: A Randomized Controlled Trial
}

\author{
Isabell Englert ${ }^{a} \quad$ Anja Bosy-Westphal ${ }^{b}$ Stephan C. Bischoff ${ }^{c}$ \\ Kathrin Kohlenberg-Müller ${ }^{\mathrm{a}}$

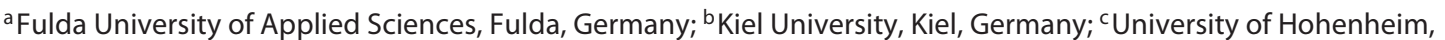 \\ Stuttgart, Germany
}

\section{Keywords}

Weight loss · Protein · Fat-free mass · Resting energy

expenditure $\cdot$ Physical function

\begin{abstract}
Introduction: Weight loss in old age increases the risk of sarcopenia caused by the age-related reduction of fat-free mass (FFM). Due to the strong correlation between FFM and resting energy expenditure (REE), the maintenance of this must also be considered. Besides, the physical function (PF) must be maintained. Objective: The impact of protein intake on changes in FFM, REE, and PF during weight loss in overweight postmenopausal women was investigated. Methods: Fifty-four postmenopausal women (BMI 30.9 \pm 3.4 ; age $59 \pm 7$ years) were randomized into 2 groups receiving energy-restricted diets with either $0.8 \mathrm{~g}$ (normal protein; NP) or $1.5 \mathrm{~g}$ protein/kg body weight (high protein; HP) for 12 weeks, followed by a 6-month follow-up phase with an ad libitum food intake. FFM, REE, and PF (strength, endurance, and balance) were measured at baseline, after weight loss, and after follow-up. Results: Forty-six women completed the weight loss intervention and 29 were followed up. The weight loss was $-4.6 \pm 3.6 \mathrm{~kg}(\mathrm{HP})$ and $-5.2 \pm 3.4 \mathrm{~kg}$ (NP; both $p<0.001)$ and the weight regain during follow-up was $1.3 \pm 2.8 \mathrm{~kg}(\mathrm{HP}$;
\end{abstract}

$p=0.03)$ and $0.4 \pm 2.5 \mathrm{~kg}(\mathrm{NP} ; p=0.39)$, with no differences between groups. Similar decreases in FFM $(-0.9 \pm 1.1$ [HP] vs. $-1.0 \pm 1.3 \mathrm{~kg}[\mathrm{NP}])$ and REE (-862 \pm 569 [HP] vs. $-1,000 \pm 561$ $\mathrm{kJ}[\mathrm{NP}]$; both $p<0.001)$ were observed in both groups. During follow-up, no changes in FFM were detected in either group, whereas in the NP group the REE increased again $(+138 \pm 296 ; p=0.02)$. The main determinants of FFM loss were the energy deficit and the speed of weight loss. In the NP group, the Short Physical Performance Battery score improved with weight loss $(+0.6 \pm 0.8 ; p<0.001)$ and handgrip strength decreased $(-1.7 \pm 3.4 \mathrm{~kg} ; p<0.001)$, whereas no changes were observed in the HP group. Conclusions: An HP weight-loss diet without exercise had no impact on preservation of FFM and REE but may help to maintain muscle strength in postmenopausal women.

(c) 2021 The Author(s)

Published by S. Karger AG, Basel

\section{Introduction}

In Germany $61 \%$ of women aged 50-59 years are overweight and $27 \%$ are obese, and the prevalence increases with age [1]. Obesity can be associated with reduced muscle mass and strength, especially in older people [2]; this is known as "sarcopenic obesity" [3] and it increases the karger@karger.com www.karger.com/ofa

Karger $\frac{1}{\%}$

BOPEN ACCESS
(C) 2021 The Author(s)

Published by S. Karger AG, Basel

This is an Open Access article licensed under the Creative Commons Attribution-NonCommercial-4.0 International License (CC BY-NC) (http://www.karger.com/Services/OpenAccessLicense), applicable to the online version of the article only. Usage and distribution for commercial purposes requires written permission.
Correspondence to:

Isabell Englert, isabell-englert@web.de 
risk of low physical function (PF) [4]. Weight reduction in particular is also associated with a loss of lean muscle mass [4] and thus increases the risk of sarcopenia in older adults [3]. The combination of a higher prevalence of obesity and sarcopenia in women increases the genderspecific risk [5, 6]. Nonetheless, intentional weight loss can improve the PF $[7,8]$ and metabolic profile $[7,9,10]$. Therefore, suitable scientifically based dietary recommendations for weight reduction in older people should be generated to minimize the risk of FFM loss. Previous studies have shown that weight loss with a high-protein (HP) diet ( 1.4 vs. $0.8 \mathrm{~g} / \mathrm{kg}$ protein/day) may help to preserve FFM in adults aged $20-80$ years [9-11]. Two studies in older adults (age 50-70 years) showed a better preservation of FFM with a higher protein intake ( 30 vs. $15 \%$ protein of the total energy intake or 1.11 vs. $0.85 \mathrm{~g} / \mathrm{kg}$ ) during a weight loss of about $9 \mathrm{~kg}$ in 20 weeks [12] and $3 \mathrm{~kg}$ in 13 weeks, respectively, but the latter with an exercise program [13]. In contrast, some other studies did not show an improved preservation of lean mass with a higher protein content in a weight loss diet (10 weeks to 6 months) in older people (age $>55$ years; $0.8-1.0$ vs. $1.2-$ $1.7 \mathrm{~g} / \mathrm{kg}$ protein) $[14,15]$, one of them with exercise [16]. Also after bariatric surgery, which is accompanied by a significant loss of muscle mass, an HP diet does not prevent muscle mass loss [17]. The designs of the available studies (intervention period, gender, age, and with and without exercise), however, are very heterogeneous and do not allow clear conclusions. In contrast to middleaged adults, the presence of significant effects in older persons is missing. Furthermore, not all of them were randomized controlled studies (RCT) and only 1 of them had a blinded design. For this issue, no double blinded RCT in postmenopausal women is available.

Due to the association between FFM and resting energy expenditure (REE) [18], the weight loss-induced decrease in FFM also impairs REE and thus increases the risk of weight regain [19]. In addition, Porter Starr et al. [7] showed that a high protein intake ( $1.2 \mathrm{vs.} 0.8 \mathrm{~g} / \mathrm{kg}$ ) can help to improve or maintain PF during weight reduction even without physical exercise in adults aged $>60$ years.

The aim of this study was to investigate the effect of an HP diet without exercise during weight loss on changes in FFM, REE, and PF in overweight postmenopausal women. We hypothesized that postmenopausal women on an HP diet experience less of a decrease in muscle mass, REE, and PF during weight reduction compared to the control group. The primary outcome was FFM. Secondary outcomes included REE and PF.

\section{Subjects and Methods}

This study was conducted at the Department Nutritional, Food and Consumer Sciences of the Fulda University of Applied Sciences. Fifty-four women were recruited via local flyers or advertisement and randomized in a 1: 1 ratio to 2 parallel arms, i.e., a normal protein group (NP; $0.8 \mathrm{~g} / \mathrm{kg}$ body weight/day, based on current recommendations at the time of the start of the study [20]) and a HP group (HP; $1.5 \mathrm{~g} / \mathrm{kg}$ ). In order to be included in this study, participants aged $\geq 50$ years and with a BMI $\geq 30$ or $\geq 27$ and waist circumference $>88 \mathrm{~cm}$ had to be able to walk without auxiliaries, climb 10 stairs, and to do their typical daily activities. The estimated physical activity level (PAL) had to be $<1.8$ and constant during the last 3 months. All women provided written informed consent before randomization. Participants were excluded for the following reasons: $\mathrm{BMI} \geq 35$; creatinine $>2.0 \mathrm{mg} / \mathrm{dL}$; weight change $> \pm 5 \%$ in the last 6 months; type 2 diabetes; thyroid disease; medication (steroids, diuretics, thyroid drugs, statins, weight loss medication, and $\beta$-blockers); participation in other weight reduction programs; kidney, heart, or liver failure; protein supplementation during the last 3 months; neurological disorders; electronic implants; active prostheses; life-sustaining electronic devices; aversion or allergy to whey products (self-information).

A 1: 1 randomization with random block sizes between 12 and 16 was done by an independent computer scientist. A computergenerated list with random numbers was used and, relevant to block size, allocated to a ranking. The study participants and study staff were blinded to the allocation into groups. Annulment of blinding was done after completion of the study.

The intervention period of 12 weeks included 4 nutrition training sessions for both groups separately and telephone interviews to enhance compliance. The weight loss period was followed by an ad libitum food intake for 6 months without intervention (followup phase). The primary outcome was the change in FFM after the 12 -week weight loss period, and secondary outcome parameters were changes in REE and PF after 12 weeks. An outline of the study protocol is given in Figure 1.

The individual total energy expenditure was calculated as the measured REE multiplied by the PAL (estimated based on a 7-day activity diary) and reduced by about $3,139 \mathrm{~kJ}(750 \mathrm{kcal})$ per day for both groups to derive the individual prescription of energy intake. At baseline, dietary history gave information about the preferences and aversions toward food. The individually prescribed energy intake was translated into a meal plan as follows: the participants received a meal replacement (Precon shake; Darmstadt, Germany; $1,565 \mathrm{~kJ}$ [ $374 \mathrm{kcal}$ ], $12.2 \mathrm{~g}$ fat, $8.7 \mathrm{~g}$ carbohydrates, and $46.6 \mathrm{~g}$ protein per $100 \mathrm{~g}) 2$ times a day prepared with $300 \mathrm{~mL}$ milk ( $1.5 \%$ fat). The third meal and/or snack should be chosen from the individual diet plan. The shakes of the HP group were fortified with whey powder (Primal State, Berlin, Germany; 1,686 kJ [403 kcal], $8.5 \mathrm{~g}$ fat, $5.7 \mathrm{~g}$ carbohydrates, $76 \mathrm{~g}$ protein per $100 \mathrm{~g}$ ) to achieve the target amount of protein. The differences in protein intake were compensated for by the caloric load on carbohydrate intake in the control group. There were no restrictions of timing for meal intake. For the first 3 weeks, women obtained individual daily meal plans and at each following session they received 10 new plans. Two dietitians were trained in advance to ensure the same content and methods were used during training in all of the groups. Participants had to keep a food diary (after the first and third training sessions for 7 consecutive days) and food checklists (on the remaining days). 
Fig. 1. Schematic representation of this study.

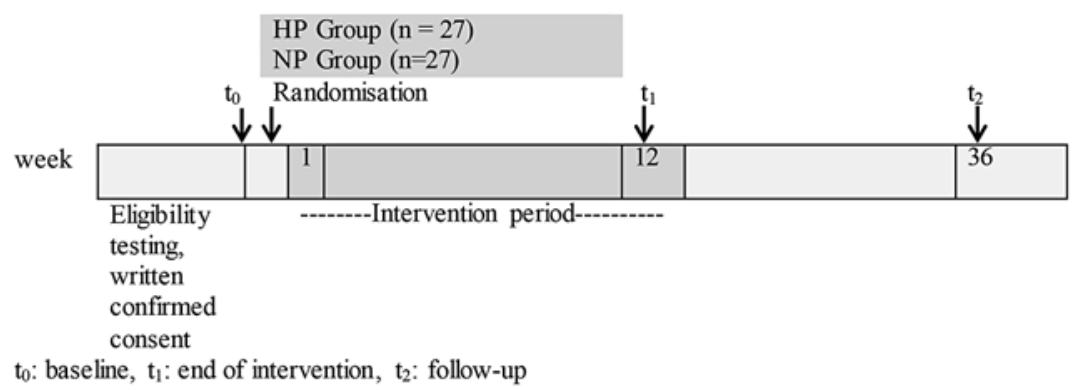

$t_{0}$ : baseline, $t_{1}$ : end of intervention, $t_{2}$ : follow-up
Anthropometry, REE, and PF measurements were done by a trained nutritionist at baseline $\left(\mathrm{t}_{0}\right)$, after the intervention time $\left(\mathrm{t}_{1}\right)$, and after the ad libitum phase $\left(t_{2}\right)$.

REE was measured under standardized conditions after an overnight fast in the morning using indirect calorimetry (Quark RMR; Cosmed, Germany). Before REE was recorded for at least 20 $\mathrm{min}$, the subjects lay supine on a patient couch for $30 \mathrm{~min}$. The first 10 min of data were discarded. The calorimeter was calibrated each morning according to the manufacturer's guidelines.

Body height was determined barefoot in a standing position using a stadiometer (seca 274; seca GmbH, Hamburg, Germany). Body weight was recorded after an overnight fast barefoot in light, everyday clothing using a bioelectrical impedance analysis (seca medical body composition analyzer; seca mBCA 515/514). FFM and fat mass were measured with the patient in a standing position and barefoot, with 8 electrodes for both hands and feet (seca mBCA 515/514), under standardized conditions, with an empty bladder, minimal fasting time, and abstinence from strenuous physical exercise of $8 \mathrm{~h}$, respectively. Waist circumference was measured in an upright position between the lower rib and the iliac crest using a seca tapeline.

The Short Physical Performance Battery (SPPB) [21] was used to measure 3 categories, i.e., balance, gait speed, and strength. Women had to walk a distance of $400 \mathrm{~m}$ as fast as they could without running. A Jamar hand dynamometer was used to measure isometric hand grip strength using the standardized Southampton protocol [22].

Fasting blood samples were analyzed at the Fulda clinic for concentrations of serum urea nitrogen in order to assess differences in protein intake among study groups. To assess kidney and liver function, the glomerular filtration rate and creatinine and albumin levels were measured.

Target weight loss was calculated using a body weight planner [23] based on weight, sex, age, height, PAL, \% fat mass, \% calories from carbohydrates and REE and compared with the actual weight loss for evaluation of compliance.

To detect differences in changes in FFM with weight loss between groups, 27 women per group were needed at a significance level of $\alpha=0.05$ and a power of $80 \%$. This calculation was based on an effect size of 0.69 and an SD of 1.5 [10]. Data are presented as means \pm SD. An intention-to-treat analysis with the last observation carried forward for all of the participants and a per protocol analysis for the participants who completed the study were conducted. Between-subject analyses served to compare outcomes be-
Table 1. Baseline characteristics of the study participants by treatment group

\begin{tabular}{lccc}
\hline & $\begin{array}{l}\text { HP group } \\
(n=27)\end{array}$ & $\begin{array}{l}\text { NP group } \\
(n=27)\end{array}$ & $p$ value $^{\mathrm{a}}$ \\
\hline Age, years & $59.0 \pm 6$ & $58.7 \pm 6$ & 0.566 \\
PAL & $1.4 \pm 0.1$ & $1.4 \pm 0.1$ & 0.377 \\
Body weight, kg & $85.1 \pm 8.6$ & $84.9 \pm 9.8$ & 0.929 \\
BMI & $30.5 \pm 2.8$ & $31.3 \pm 4.0$ & 0.439 \\
FFM, kg & $46.8 \pm 6.9$ & $46.7 \pm 5.0$ & 0.940 \\
Fat mass, kg & $38.3 \pm 5.6$ & $38.2 \pm 6.9$ & 0.947 \\
Waist circumference, cm & $98.2 \pm 6.9$ & $98.4 \pm 10.8$ & 0.878 \\
REE, kJ (kcal) & $7,174 \pm 707$ & $7,082 \pm 837$ & \\
& $(1,714 \pm 169)$ & $(1,692 \pm 200)$ & 0.665 \\
SPPB score & $9.4 \pm 1.1$ & $9.9 \pm 1.0$ & 0.119 \\
Handgrip strength, kg & $28.7 \pm 7.2$ & $29.0 \pm 4.9$ & 0.652 \\
400-m walk, min:s & $4: 10 \pm 0: 33$ & $04: 11 \pm 0: 31$ & 0.692 \\
Urea, mg/dL & $33.2 \pm 7.6$ & $27.5 \pm 5.6$ & 0.003 \\
\hline
\end{tabular}

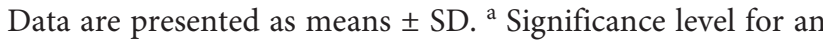
independent $t$ test.

tween groups over time (independent $t$ test), and within-subject analyses were used to measure effects over time (dependent $t$ test, $t_{0}-t_{1}, t_{1}-t_{2}$ ). An analysis of variance (ANOVA), if applicable with a Greenhouse-Geisser correction, was also done with Bonferronicorrected pairwise comparisons to detect specific time differences. The significance level was set at $\alpha=0.05$.

\section{Results}

After screening 197 subjects for eligibility (October 2016 to April 2017), 54 women were randomized into 2 groups (Fig. 2). Eight women dropped out during the intervention because of adverse events not related to this study $(n=5)$ and nonacceptance of the shakes $(n=3)$. Twenty-nine women completed this study, including the 
Table 2. Dietary energy and macronutrient intake data in the first and third quarters of the intervention

\begin{tabular}{llllll}
\hline & & Energy, kJ/day (kcal/day) & $\begin{array}{l}\text { Protein, } \\
\text { g/day }\end{array}$ & $\begin{array}{l}\text { Carbohydrates, } \\
\text { g/day }\end{array}$ & $\begin{array}{l}\text { Fat, } \\
\text { g/day }\end{array}$ \\
\hline Intervention weeks 1-3 & HP $(n=21)$ & $6,048 \pm 695(1,445 \pm 166)$ & $114 \pm 16$ & $127 \pm 22$ & $45 \pm 8$ \\
& NP $(n=25)$ & $5,249 \pm 917(1,254 \pm 219)^{*}$ & $61 \pm 9^{*}$ & $134 \pm 37$ & $46 \pm 14$ \\
\hline Intervention weeks 7-9 & HP $(n=20)$ & $6,082 \pm 992(1,453 \pm 237)$ & $112 \pm 22$ & $126 \pm 20$ & $48 \pm 12$ \\
& NP $(n=25)$ & $5,584 \pm 1101(1,334 \pm 263)$ & $65 \pm 11^{*}$ & $138 \pm 33$ & $51 \pm 12$ \\
\hline Mean & HP $(n=20)$ & $6,065 \pm 737(1,449 \pm 176)$ & $113 \pm 17$ & $127 \pm 18$ & $46 \pm 9$ \\
& NP $(n=25)$ & $5,417 \pm 904(1,294 \pm 216)^{*}$ & $63 \pm 9^{*}$ & $136 \pm 29$ & $48 \pm 11$ \\
\hline
\end{tabular}

Data are presented as means $\pm \mathrm{SD}$. ${ }^{*}$ Significant difference between groups $(p<0.05$, independent $t$ test).

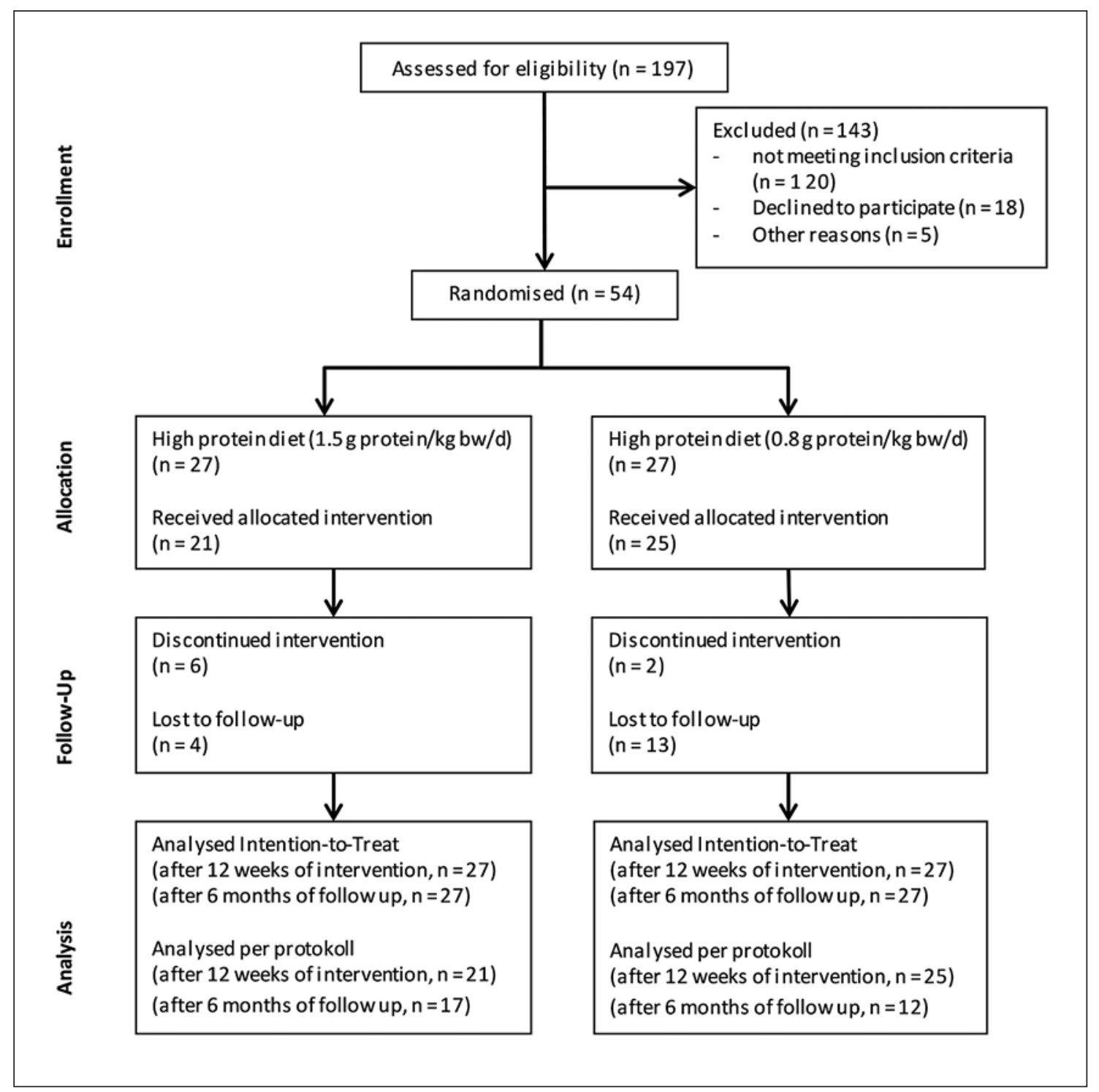

Fig. 2. CONSORT flow chart of the study design. 
Table 3. Absolute changes in body weight, FFM, fat mass, waist circumference, REE, SPPB, 400-m walk, and handgrip with weight loss and follow-up by treatment group

\begin{tabular}{|c|c|c|c|c|c|}
\hline & \multicolumn{2}{|l|}{ HP group } & \multicolumn{2}{|l|}{ NP group } & \multirow{2}{*}{$\begin{array}{l}p \text { value } \\
(\mathrm{HP} \times \mathrm{NP})^{\mathrm{b}}\end{array}$} \\
\hline & mean \pm SD & $p$ value $^{\mathrm{a}}$ & mean \pm SD & $p$ value ${ }^{\mathrm{a}}$ & \\
\hline \multicolumn{6}{|l|}{ Body weight, kg } \\
\hline Baseline & $85.1 \pm 8.6$ & & $84.9 \pm 9.8$ & & \\
\hline Change at $t_{1}$ & $-4.6 \pm 3.6$ & $<0.001$ & $-5.2 \pm 3.4$ & $<0.001$ & 0.538 \\
\hline Change at $t_{2}$ & $+1.3 \pm 2.8$ & 0.028 & $+0.4 \pm 2.5$ & 0.392 & 0.253 \\
\hline \multicolumn{6}{|l|}{ FFM, kg } \\
\hline Baseline & $46.8 \pm 6.9$ & & $46.7 \pm 5.0$ & & \\
\hline Change at $t_{1}$ & $-0.9 \pm 1.1$ & $<0.001$ & $-1.0 \pm 1.3$ & $<0.001$ & 0.575 \\
\hline Change at $t_{2}$ & $+0.4 \pm 1.4$ & 0.146 & $-0.0 \pm 1.6$ & 0.890 & 0.181 \\
\hline \multicolumn{6}{|l|}{$\mathrm{FM}, \mathrm{kg}$} \\
\hline Baseline & $38.3 \pm 5.6$ & & $38.2 \pm 6.9$ & & \\
\hline Change at $t_{1}$ & $-3.8 \pm 2.8$ & $<0.001$ & $-4.0 \pm 2.7$ & $<0.001$ & 0.718 \\
\hline Change at $t_{2}$ & $+0.9 \pm 2.1$ & 0.040 & $+0.3 \pm 2.1$ & 0.478 & 0.315 \\
\hline \multicolumn{6}{|c|}{$\begin{array}{l}\text { Waist circumference, } \\
\mathrm{cm}\end{array}$} \\
\hline Baseline & $98.2 \pm 6.9$ & & $98.4 \pm 10.8$ & & \\
\hline Change at $t_{1}$ & $-6.5 \pm 4.2$ & $<0.001$ & $-7.2 \pm 4.0$ & $<0.001$ & 0.669 \\
\hline Change at $t_{2}$ & $-0.6 \pm 4.6$ & 0.525 & $-0.3 \pm 2.4$ & 0.522 & 0.756 \\
\hline \multicolumn{6}{|l|}{ REE, kJ (kcal) } \\
\hline Baseline & $7,175 \pm 707(1,714 \pm 169)$ & & $7,083 \pm 837(1,692 \pm 200$ & & \\
\hline Change at $t_{1}$ & $-862 \pm 569(-206 \pm 136)$ & $<0.001$ & $-1,000 \pm 561(-239 \pm 13$ & 4) $<0.001$ & 0.369 \\
\hline Change at $t_{2}$ & $+25 \pm 276(+6 \pm 66)$ & 0.650 & $+138 \pm 297(+33 \pm 71)$ & 0.022 & 0.148 \\
\hline \multicolumn{6}{|l|}{ SPPB, score } \\
\hline Baseline & $9.4 \pm 1.1$ & & $9.9 \pm 1.0$ & & \\
\hline Change at $t_{1}$ & $+0.4 \pm 0.9$ & 0.051 & $+0.6 \pm 0.8$ & $<0.001$ & 0.463 \\
\hline Change at $t_{2}$ & $+0.4 \pm 0.7$ & 0.015 & $+0.1 \pm 0.4$ & 0.327 & 0.073 \\
\hline \multicolumn{6}{|c|}{ 400-m walk, min:s } \\
\hline Baseline & $4: 10 \pm 0: 33$ & & $04: 11 \pm 0: 31$ & & \\
\hline Change at $t_{1}$ & $-0: 00 \pm 0: 07$ & 0.876 & $-0: 05 \pm 0: 12$ & 0.045 & 0.281 \\
\hline Change at $t_{2}$ & $+0: 01 \pm 0: 19$ & 0.630 & $+0: 02 \pm 0: 26$ & 0.636 & 0.919 \\
\hline \multicolumn{6}{|l|}{ Handgrip, kg } \\
\hline Baseline & $28.7 \pm 7.2$ & & $29.0 \pm 4.9$ & & \\
\hline Change at $t_{1}$ & $+0.1 \pm 2.6$ & 0.798 & $-1.6 \pm 3.3$ & $<0.001$ & 0.041 \\
\hline Change at $t_{2}$ & $+0.1 \pm 1.9$ & 0.864 & $-0.2 \pm 1.5$ & 0.493 & 0.577 \\
\hline
\end{tabular}

${ }^{a}$ Significance level for dependent $t$ test within-group changes $\left(t_{0}-t_{1} ; t_{1}-t_{2}\right)$. ${ }^{b}$ Significance level for independent $t$ test between-group changes.

follow-up. Baseline characteristics are shown in Table 1. There were no significant differences in baseline characteristics between the groups, except for a higher serum urea in the HP group $(p=0.003)$. During the intervention, the HP group consumed on average $113 \pm 17$ g protein $(1.4 \pm 0.1 \mathrm{~g} / \mathrm{kg})$ and the NP group $63 \pm 9 \mathrm{~g}$ protein per day $(0.8 \pm 0.1 \mathrm{~g} / \mathrm{kg}$; Table 2$)$. The results of the per protocol analysis were not different from the results of the intention-to-treat analysis. So, only the results of the intentionto-treat analysis are presented.

Protein Intake and Preservation of FFM with Weight Loss
No differences were found between the predicted and real energy deficits calculated by the body weight planner [23]; the values were: $-179 \pm 1,117 \mathrm{~kJ} /$ day $(-43 \pm 267$ $\mathrm{kcal} /$ day; $p=0.468)$ in the HP group and $105 \pm 1,326 \mathrm{~kJ} /$ day $(25 \pm 317 \mathrm{kcal} /$ day; $p=0.686)$ in the NP group. However, there was a difference between the real and predicted changes in FFM in both groups $(1.7 \pm 1.1 \mathrm{~kg}$; $p<$ 0.001 [HP] and $1.4 \pm 1.4 \mathrm{~kg} ; p<0.001$ [NP]). The recorded total energy intake was higher in the HP group than in the NP group. The deviation of the recorded energy intake from the planned one was smaller in the HP 


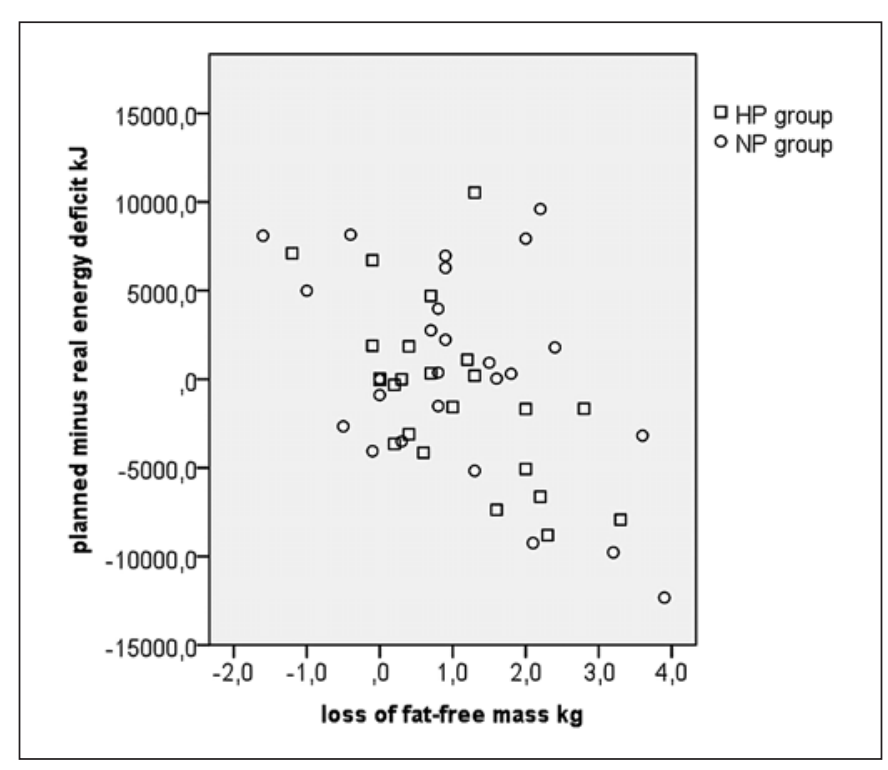

Fig. 3. Loss of lean mass vs. the planned minus real energy deficit. Correlation coefficient of the HP group: $r=-0.58 ; p=0.002$. Correlation coefficient of the NP group: $r=-0.45 ; p=0.019$. Correlation coefficient overall: $r=-0.49 ; p<0.001$.

group than in the NP group, but this was not significant $(p=0.44)$.

The intervention led to a similar weight loss in both groups (Table 3), which was still significant at the end of the follow-up phase. ANOVA showed no within-group differences during follow-up ( $\mathrm{t}_{1}$ vs. $\left.\mathrm{t}_{2} ; p=0.074\right)$ and no between-group differences $\left(\mathrm{t}_{1}: p=0.538, \mathrm{t}_{2}: p=0.264\right.$, and over time: $p=0.343)$. A higher energy deficit and a higher weight loss, respectively, were associated with a higher loss of FFM $(r=-0.49 ; p<0.001, r=0.78 ; p<0.001$; Fig. 3 , $4)$. No significant between-group differences were observed for changes in FFM, fat mass, and waist circumference at $t_{1}$ and $t_{2}$ (all $\left.p>0.05\right)$. The decrease in FFM did not correlate with protein consumption (in $\mathrm{g} / \mathrm{kg}$ FFM). Thirty-nine percent of the variance in FFM loss was explained by the speed of weight loss during the first 3 weeks and the energy deficit.

REE showed a similar decrease in weight loss in both groups ( $p=0.369)$. At $t_{2}$ significant reductions remained without a group effect $(p=0.871)$. After adjusting REE for FFM using regression analysis, REE remained reduced with weight loss $(p<0.001)$.

The SPPB revealed improvements with weight loss (HP: $p=0.051 ; \mathrm{NP}: p<0.001$ ) and follow-up (both groups: $p<0.001$ ), with no differences between groups. Results from the 400-m test remained unchanged in both groups

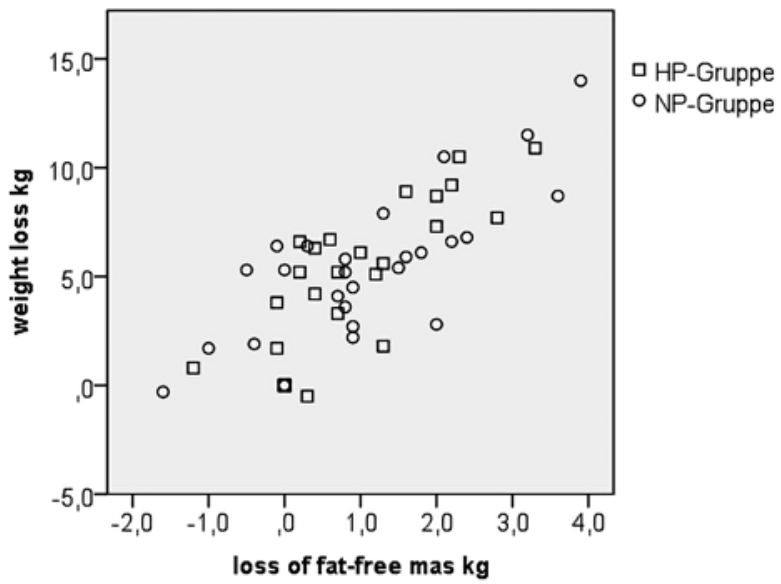

Fig. 4. Loss of lean mass vs. weight loss. Correlation coefficient of the HP group: $r=-0.80 ; p<0.001$. Correlation coefficient of the NP group: $r=-0.78 ; p<0.001$. Correlation coefficient overall: $r=$ $-0.78 ; p<0.001$.

with weight loss and follow-up. Handgrip strength remained unchanged with weight loss $(p=0.798)$ in the HP group but decreased in the NP group $(p=0.019)$. This group effect was significant $(p=0.041)$.

\section{Discussion}

The hypothesis that an increased protein intake without exercise during weight loss in postmenopausal women promotes the preservation of FFM was not confirmed. Reasons for the lack of group effects in the weight loss phase may be the large difference between the predicted and real FFM changes. However, compliance was the same in both groups, so there is no systematic bias. Limitations in measurement precision and the validity of the bioelectrical impedance analysis during the instable phase of weight loss, i.e., because of a decrease in FFM hydration [24], would be another reason, though there needs to be at least a minimal difference, which could not be shown. Furthermore, our results are consistent with studies that used dual X-ray absorptiometry or air displacement plethysmography (Table 4); Backx et al. [15] used 1.7 vs. $0.9 \mathrm{~g} /$ $\mathrm{kg}$ protein in their 12 -week RCT and achieved a weight loss of about $9 \mathrm{~kg}$ in 61 subjects aged $>55$ years, without differences in FFM loss between the groups. Similarly, in 
Table 4. Intervention studies on the effects of protein intake during weight loss on FFM

\begin{tabular}{|c|c|c|c|c|c|}
\hline Study & Proband characteristics & Protein intake & Energy restriction & Weight reduction & FFM change \\
\hline $\begin{array}{l}\text { Johnston et al. } \\
\text { [27] }\end{array}$ & $\begin{array}{l}21 \text { subjects } \\
\text { Male and female } \\
\text { Age } 20-75 \text { years } \\
\text { BMI } 20-42\end{array}$ & $\begin{array}{l}125 \mathrm{~g} / \text { day } \\
80 \mathrm{~g} / \text { day }\end{array}$ & $\begin{array}{l}-500 \mathrm{kcal} / \text { day from the TEE (calculated } \\
\text { using a formula) } \\
6 \text { weeks }\end{array}$ & $\begin{array}{l}-2.3 \pm 2.9 \mathrm{~kg}(\mathrm{P}) \\
-2.0 \pm 1.8 \mathrm{~kg}(\mathrm{C}) \\
\text { Group difference n.s. }\end{array}$ & $\begin{array}{l}+1.5 \pm 3.8 \mathrm{~kg}(\mathrm{P}) \\
-0.5 \pm 1.5 \mathrm{~kg}(\mathrm{C}) \\
\text { Group difference } p=0.008\end{array}$ \\
\hline $\begin{array}{l}\text { Verreijen et al. } \\
\text { [13] }\end{array}$ & $\begin{array}{l}88 \text { subjects } \\
\text { Male and female } \\
\text { Age }>55 \text { years } \\
\text { BMI }>30 \text { (men) or }>28 \text { (women) and } \\
\text { waist circumference }>88 \mathrm{~cm} \text { (women) } \\
\text { or }>102 \mathrm{~cm} \text { (men) }\end{array}$ & $\begin{array}{l}1.11 \mathrm{~g} / \mathrm{kg} \mathrm{BW} / \text { day } \\
0.85 \mathrm{~g} / \mathrm{kg} \mathrm{BW} / \text { day }\end{array}$ & $\begin{array}{l}-600 \mathrm{kcal} / \text { day from the TEE (REE } \\
\text { measured by indirect calorimetry }+ \text { PAL } \\
\text { measured using a } 3 \text {-day movement diary) } \\
13 \text { weeks }\end{array}$ & $\begin{array}{l}-3.4 \pm 3.6 \mathrm{~kg} \\
-2.8 \pm 2.8 \mathrm{~kg} \\
\text { Group difference n.s. }\end{array}$ & $\begin{array}{l}\text { Appendicular muscle mass } \\
+0.4 \pm 1.2 \mathrm{~kg}(\mathrm{P}) \\
-0.5 \pm 2.1 \mathrm{~kg}(\mathrm{C}) \\
\text { Group difference } p=0.03 \\
\text { Leg muscle mass } \\
+0.3 \pm 1.2 \mathrm{~kg}(\mathrm{P}) \\
-0.6 \pm 1.8 \mathrm{~kg}(\mathrm{C}) \\
\text { Group difference } p=0.01\end{array}$ \\
\hline $\begin{array}{l}\text { Backx et al. } \\
{[15]}\end{array}$ & $\begin{array}{l}61 \text { subjects } \\
\text { Male and female } \\
\text { Age } 55-70 \text { years } \\
\text { BMI } 27-40 \text { and waist } \\
\text { circumference }>88 \mathrm{~cm} \text { (women) or } \\
>102 \mathrm{~cm} \text { (men) }\end{array}$ & $\begin{array}{l}1.7 \mathrm{~g} / \mathrm{kg} \mathrm{BW} / \text { day } \\
0.9 \mathrm{~g} / \mathrm{kg} \mathrm{BW} / \text { day }\end{array}$ & $\begin{array}{l}-25 \% \text { of the baseline energy intake } \\
\text { calculated from a validated } 177 \text {-item food } \\
\text { frequency questionnaire } \\
12 \text { weeks }\end{array}$ & $\begin{array}{l}\text { From } 92.8 \pm 11.0 \text { to } 83.9 \pm 10.1 \mathrm{~kg}(\mathrm{P}) \\
\text { From } 90.5 \pm 10.0 \text { to } 81.5 \pm 9.7 \mathrm{~kg}(\mathrm{C}) \\
\text { Group difference n.s. }\end{array}$ & $\begin{array}{l}\text { From } 54.8 \pm 12.2 \text { to } 53.1 \pm 11.4 \mathrm{~kg}(\mathrm{P}) \\
\text { From } 54.5 \pm 9.3 \text { to } 52.4 \pm 9.1 \mathrm{~kg}(\mathrm{C}) \\
\text { Group difference n.s. }\end{array}$ \\
\hline $\begin{array}{l}\text { Bales et al. } \\
{[8]}\end{array}$ & $\begin{array}{l}80 \text { women } \\
\text { Age } \geq 45 \text { years } \\
\text { BMI } \geq 30\end{array}$ & $\begin{array}{l}1.2 \mathrm{~g} / \mathrm{kg} \mathrm{BW} / \text { day } \\
0.8 \mathrm{~g} / \mathrm{kg} \mathrm{BW} / \text { day }\end{array}$ & $\begin{array}{l}-500 \mathrm{kcal} / \text { day from the TEE (calculated } \\
\text { using a formula) } \\
6 \text { months }\end{array}$ & $\begin{array}{l}-6.2 \pm 5.9 \mathrm{~kg}(\mathrm{P}) \\
-6.4 \pm 4.9 \mathrm{~kg}(\mathrm{C}) \\
\text { Group difference n.s. }\end{array}$ & $\begin{array}{l}-0.6 \pm 1.1 \mathrm{~kg}(\mathrm{P}) \\
-1.0 \pm 1.1 \mathrm{~kg}(\mathrm{C}) \\
\text { Group difference n.s. }\end{array}$ \\
\hline $\begin{array}{l}\text { Porter Starr et al. } \\
\text { [7] }\end{array}$ & $\begin{array}{l}67 \text { subjects } \\
\text { Male and female } \\
\text { Age }>60 \text { years } \\
\text { BMI } \geq 30\end{array}$ & $\begin{array}{l}1.2 \mathrm{~g} / \mathrm{kg} \mathrm{BW} / \text { day } \\
0.8 \mathrm{~g} / \mathrm{kg} \mathrm{BW} / \text { day }\end{array}$ & $\begin{array}{l}-500 \mathrm{kcal} / \mathrm{day} \\
6 \text { months }\end{array}$ & $\begin{array}{l}-8.7 \pm 7.4 \mathrm{~kg}(\mathrm{P}) \\
-7.5 \pm 6.2 \mathrm{~kg}(\mathrm{C}) \\
\text { Group difference n.s. }\end{array}$ & $\begin{array}{l}-1.1 \pm 1.5 \mathrm{~kg}(\mathrm{P}) \\
-1.8 \pm 2.9 \mathrm{~kg}(\mathrm{C}) \\
\text { Group difference n.s. }\end{array}$ \\
\hline $\begin{array}{l}\text { Layman et al. } \\
{[25]}\end{array}$ & $\begin{array}{l}130 \text { subjects } \\
\text { Male and female } \\
\text { Age } 40-56 \text { years } \\
\text { BMI }>27\end{array}$ & $\begin{array}{l}1.6 \mathrm{~g} / \mathrm{kg} \mathrm{BW} / \text { day } \\
0.8 \mathrm{~g} / \mathrm{kg} \mathrm{BW} / \text { day }\end{array}$ & $\begin{array}{l}-500 \mathrm{kcal} / \mathrm{day} \\
4 \text {-month weight reduction } \\
8 \text { months with a stable weight }\end{array}$ & $\begin{array}{l}\text { After } 4 \text { months: } \\
-8.2 \pm 0.5 \mathrm{~kg}(\mathrm{P}) \\
-7.0 \pm 0.5 \mathrm{~kg}(\mathrm{C}) \\
\text { Group difference n.s. }\end{array}$ & $\begin{array}{l}-2.6 \pm 0.2 \mathrm{~kg}(\mathrm{P}) \\
-2.4 \pm 0.2 \mathrm{~kg}(\mathrm{C}) \\
\text { Group difference n.s. }\end{array}$ \\
\hline $\begin{array}{l}\text { Flechtner-Mors et al. } \\
\text { [26] }\end{array}$ & $\begin{array}{l}110 \text { subjects } \\
\text { Male and female } \\
\text { Age } 25-70 \text { years } \\
\text { BMI } 27-45\end{array}$ & $\begin{array}{l}1.34 \mathrm{~g} / \mathrm{kg} \mathrm{BW} / \text { day } \\
0.8 \mathrm{~g} / \mathrm{kg} \mathrm{BW} / \text { day }\end{array}$ & $\begin{array}{l}-500 \text { kcal from the REE (formula) } \\
3 \text {-month weight reduction } \\
9 \text { months with a stable weight }\end{array}$ & $\begin{array}{l}\text { After } 3 \text { months: } \\
-7.4 \pm 4.6 \mathrm{~kg}(\mathrm{P}) \\
-4.8 \pm 4.0 \mathrm{~kg}(\mathrm{C}) \\
\text { Group difference } p<0.01\end{array}$ & $\begin{array}{l}\text { After } 3 \text { months } \\
-2.8 \pm 3.6 \mathrm{~kg}(\mathrm{P}) \\
-3.2 \pm 2.7 \mathrm{~kg}(\mathrm{C}) \\
\text { Group difference n.s. }\end{array}$ \\
\hline
\end{tabular}

TEE, total energy expenditure; n.s., nonsignifcant; EN\%, percentage of energy; P, protein group; C, control group; BW, body weight.

Table 5. Intervention studies on the effects of protein intake during weight loss on REE

\begin{tabular}{|c|c|c|c|c|c|}
\hline Study & $\begin{array}{l}\text { Proband } \\
\text { characteristics }\end{array}$ & Protein intake & Energy restriction & $\begin{array}{l}\text { Weight reduction, } \\
\mathrm{kg}\end{array}$ & REE change \\
\hline $\begin{array}{l}\text { Tang et al. } \\
{[10]}\end{array}$ & $\begin{array}{l}43 \text { men } \\
\text { Age }>21 \text { years } \\
\text { BMI } 25-39.9\end{array}$ & $\begin{array}{l}1.4 \mathrm{~g} / \mathrm{kg} \mathrm{BW} / \text { day } \\
0.8 \mathrm{~g} / \mathrm{kg} \mathrm{BW} / \text { day }\end{array}$ & $\begin{array}{l}-750 \mathrm{kcal} / \text { day from REE } \times 1.5 \\
\text { estimated by the Harris- } \\
\text { Benedict formula } \\
12 \text { weeks }\end{array}$ & $\begin{array}{l}-9.1 \pm 0.7(\mathrm{P}) \\
-10.6 \pm 0.6(\mathrm{C}) \\
\text { Group difference n.s. }\end{array}$ & $\begin{array}{l}-104 \pm 77 \mathrm{kcal} / \text { day } \\
\text { (P) } \\
-171 \pm 71 \mathrm{kcal} / \text { day } \\
\text { (C) } \\
\text { Group difference } \\
\text { n.s. }\end{array}$ \\
\hline $\begin{array}{l}\text { Luscombe } \\
\text { et al. [37] }\end{array}$ & $\begin{array}{l}36 \text { subjects } \\
\text { Male and female } \\
\text { Age } 34-65 \text { years } \\
\text { Hyperinsulinemia } \\
\text { BMI 27-43 }\end{array}$ & $\begin{array}{l}27 \text { EN\% } \\
16 \text { EN\% }\end{array}$ & $\begin{array}{l}-30 \% \text { from TEE (measured via } \\
\text { the bicarbonate-urea method) } \\
16 \text { weeks, thereof } 4 \text { weeks of } \\
\text { balanced energy }\end{array}$ & $\begin{array}{l}-7.9 \pm 1.1(\mathrm{P}) \\
-8.0 \pm 0.7(\mathrm{C}) \\
\text { Group difference n.s. }\end{array}$ & $\begin{array}{l}-650 \pm 171 \mathrm{~kJ} / \text { day } \\
(\mathrm{P}) \\
-780 \pm 132 \mathrm{~kJ} / \text { day } \\
(\mathrm{C}) \\
\text { Group difference } \\
\text { n.s. }\end{array}$ \\
\hline
\end{tabular}

TEE, total energy expenditure; n.s., nonsignificant; EN\%, percentage of energy; P, protein group, C, control group; BW, body weight. 
Table 6. Intervention studies on the effects of protein intake during weight loss on PF

\begin{tabular}{|c|c|c|c|c|c|}
\hline Study & $\begin{array}{l}\text { Proband charac- } \\
\text { teristics }\end{array}$ & Protein intake & Energy restriction & Weight reduction, kg & PF change \\
\hline $\begin{array}{l}\text { Porter Starr } \\
\text { et al. [7] }\end{array}$ & $\begin{array}{l}67 \text { subjects } \\
\text { Male and female } \\
\text { Age }>60 \text { years } \\
\text { BMI } \geq 30\end{array}$ & $\begin{array}{l}1.2 \mathrm{~g} / \mathrm{kg} \mathrm{BW/} \\
\text { day } \\
0.8 \mathrm{~g} / \mathrm{kg} \mathrm{BW/} \\
\text { day }\end{array}$ & $\begin{array}{l}-500 \mathrm{kcal} / \text { day } \\
6 \text { months }\end{array}$ & $\begin{array}{l}-8.7 \pm 7.4(\mathrm{P}) \\
-7.5 \pm 6.2(\mathrm{C}) \\
\text { Group difference n.s. }\end{array}$ & $\begin{array}{l}\text { SPPB: } \\
+2.4 \pm 1.7(\mathrm{P}) \\
+0.9 \pm 1.7(\mathrm{C}) \\
p=0.02 \text { group difference } \\
\text { Hand grip strength was } \\
\text { unchanged in both groups }\end{array}$ \\
\hline $\begin{array}{l}\text { Wycherley } \\
\text { et al. [43] }\end{array}$ & $\begin{array}{l}43 \text { men } \\
\text { Age } 20-45 \text { years } \\
\text { BMI 27-39.9 }\end{array}$ & $\begin{array}{l}35 \%(1.3 \mathrm{~g} / \mathrm{kg} \\
\text { BW/day) } \\
17 \%(0.8 \mathrm{~g} / \mathrm{kg} \\
\text { BW/day) }\end{array}$ & $\begin{array}{l}-7,000 \mathrm{~kJ} / \text { day } \\
12 \text { weeks }\end{array}$ & $\begin{array}{l}-10.7 \pm 5.3(\mathrm{P}) \\
-8.7 \pm 3.5(\mathrm{C}) \\
\text { Group difference n.s. }\end{array}$ & $\begin{array}{l}\text { Hand grip strength: } \\
-1.9 \pm 4.2 \mathrm{~kg}(\mathrm{P}) \\
-1.4 \pm 4.1 \mathrm{~kg}(\mathrm{C}) \\
\text { Group differences n.s. } \\
\text { Isometric knee strength: } \\
+16.8 \pm 35.2(\mathrm{P}) \\
+11.5 \pm 36.8(\mathrm{C}) \\
\text { Group difference n.s. }\end{array}$ \\
\hline $\begin{array}{l}\text { Beavers et } \\
\text { al. [39] }\end{array}$ & $\begin{array}{l}69 \text { subjects } \\
\text { Male and female } \\
\text { Age } 65-79 \text { years } \\
\text { BMI } 30-40\end{array}$ & $\begin{array}{l}1.2 \mathrm{~g} / \mathrm{kg} \text { BW/ } \\
\text { day } \\
\text { (weight loss) } \\
0.8 \mathrm{~g} / \mathrm{kg} \mathrm{BW/} \\
\text { day } \\
\text { (weight stable) }\end{array}$ & $\begin{array}{l}\text {-500 kcal/day } \\
\text { Weight loss group } \\
\text { Stable-weight group }\end{array}$ & $\begin{array}{l}-6.6 \pm 0.4 \text { (weight loss) } \\
-0.2 \pm 0.5 \text { (stable } \\
\text { weight) }\end{array}$ & $\begin{array}{l}\text { Gait speed: } \\
+0.01(-0.02 \text { to } 0.04) \mathrm{m} / \mathrm{s} \\
\text { (weight loss) } \\
-0.02(-0.05 \text { to } 0.01) \mathrm{m} / \mathrm{s} \\
\text { (weight stable) } \\
\text { Group difference n.s. }\end{array}$ \\
\hline
\end{tabular}


Table 6 (continued)

\begin{tabular}{|c|c|c|c|c|c|}
\hline Study & $\begin{array}{l}\text { Proband charac- } \\
\text { teristics }\end{array}$ & Protein intake & Energy restriction & Weight reduction, $\mathrm{kg}$ & PF change \\
\hline $\begin{array}{l}\text { Verreijen } \\
\text { et al. [16] }\end{array}$ & $\begin{array}{l}100 \text { subjects } \\
\text { Male and female } \\
\text { Age } 55-80 \text { years } \\
\mathrm{BMI} \geq 28 \text { (men) or } \\
>25 \text { (women) and } \\
\text { waist circumfer- } \\
\text { ence }>88 \mathrm{~cm} \\
\text { (women) or }>102 \\
\mathrm{~cm} \text { (men) }\end{array}$ & $\begin{array}{l}1.3 \mathrm{~g} / \mathrm{kg} \mathrm{BW} / \\
\text { day } \\
0.8 \mathrm{~g} / \mathrm{kg} \text { BW/ } \\
\text { day }\end{array}$ & $\begin{array}{l}\text { - } 600 \mathrm{kcal} / \text { day from } \\
\text { TEE (REE measured } \\
\text { by indirect calorimetry } \\
+ \text { PAL measured by a } \\
3 \text {-day movement di- } \\
\text { ary) } \\
10 \text { weeks }\end{array}$ & $\begin{array}{l}-2.1 \pm 3.6(\mathrm{P}) \\
-1.7 \pm 1.8(\mathrm{C}) \\
-2.6 \pm 2.9 \text { (exercise) } \\
-2.0 \pm 2.2 \text { (exercise } \\
+\mathrm{P})\end{array}$ & $\begin{array}{l}\text { Hand grip strength: } \\
-1.7 \pm 6.5 \mathrm{~kg}(\mathrm{P}) \\
+1.8 \pm 6.6 \mathrm{~kg}(\mathrm{C}) \\
-1.8 \pm 11.6 \mathrm{~kg} \text { (exercise) } \\
-2.0 \pm 6.0 \mathrm{~kg} \text { (exercise }+\mathrm{P}) \\
\text { Protein } \times \text { exercise interac- } \\
\text { tion } p=0.03 \\
4-\mathrm{m} \text { walking speed: } \\
+0.08 \pm 0.26 \mathrm{~m} / \mathrm{s}(\mathrm{P}) \\
+0.13 \pm 0.24 \mathrm{~m} / \mathrm{s}(\mathrm{C}) \\
+0.08 \pm 0.13 \mathrm{~m} / \mathrm{s}(\text { exercise }) \\
+0.20 \pm 0.24 \mathrm{~m} / \mathrm{s}(\text { exercise } \\
+\mathrm{P}) \\
\text { Protein } \times \text { exercise } \\
\text { interaction } p=0.045 \\
400-\mathrm{m} \text { walking speed: } \\
+0.07 \pm 0.1 \mathrm{~m} / \mathrm{s}(\mathrm{P}) \\
+0.04 \pm 0.15 \mathrm{~m} / \mathrm{s}(\mathrm{C}) \\
+0.07 \pm 0.07 \mathrm{~m} / \mathrm{s}(\text { exercise }) \\
+0.08 \pm 0.15 \mathrm{~m} / \mathrm{s}(\text { exercise } \\
+\mathrm{P}) \\
\text { Group differences n.s. } \\
\text { Chair rising test: } \\
-1.6 \pm 1.7 \mathrm{~s}(\mathrm{P}) \\
-1.6 \pm 2.1 \mathrm{~s}(\mathrm{C}) \\
-1.0 \pm 2.7 \mathrm{~s}(\text { exercise }) \\
-1.4 \pm 2.7 \mathrm{~s}(\text { exercise }+\mathrm{P}) \\
\text { Group differences } \mathrm{n} . \mathrm{s} .\end{array}$ \\
\hline
\end{tabular}

EE, total energy expenditure; n.s., nonsignificant; EN\%, percentage of energy; P, protein group, C, control group; BW, body weight.

the POWR-UP study, 60 women aged $>45$ years were randomized to either an HP group $(1.3 \mathrm{~g} / \mathrm{kg})$ or an NP group $(0.8 \mathrm{~g} / \mathrm{kg})$ and achieved a weight loss of $6 \%$ within 6 months, without group effects on FFM loss [8]. In addition, in the MEASURE-UP study, equal losses of FFM were observed in 67 older subjects ( $>60$ years) over a 6-month period of weight loss regardless of the protein amount (0.8 vs. $1.2 \mathrm{~g} / \mathrm{kg}$ ) [7]. Layman et al. [25] also achieved similar FFM losses after 4 months of weight loss in 130 subjects (age $40-56$ years) with either 0.8 or $1.6 \mathrm{~g} /$ $\mathrm{kg}$ protein. In a study with 110 adults (age $25-70$ years) a significant greater weight loss but without differences in FFM loss was observed after 3 months of weight loss and after 9 months of weight maintenance in the HP group (0.8 vs. $1.34 \mathrm{~g} / \mathrm{kg}$ ) [26]. Also a study on bariatric patients (age 18-65 years) showed no better preservation of lean mass after a 6-month weight loss follow-up with a high protein intake ( $25.4 \pm 3.7$ vs. $15.8 \pm 4.4 \%$ of the energy in- take; $p<0.001$ ) [17]. Other studies, however, have reported contradictory findings (Table 4). Verreijen et al. [13] reported preservation of appendicular muscle mass in 88 obese adults aged $>55$ years on a 13-week high-whey protein hypocaloric diet ( 1.1 vs. $0.85 \mathrm{~g} / \mathrm{kg})$, which was confirmed in a 6-week study on adults (age 20-75 years) with insulin resistance ( 15 vs. $23 \%$ of energy intake) [27]. A recent meta-analysis concluded improved maintenance of FFM during weight loss without exercise in older adults (mean $>50$ years) consuming an HP diet $(\geq 25 \%$ of the total energy or $\geq 1.0 \mathrm{~g} / \mathrm{kg}$ ) for a time of 8-104 weeks (mean 25 weeks) [28]. Nevertheless, the included studies had different primary outcomes (e.g., bone density, IGF level, and cardiovascular risk factors) and were very heterogeneous. Only 4 or 2 of 20 articles provided details on randomization or blinding procedures. In addition, certain studies were considered as 2 separate studies, which may have artificially increased the protein effect. Only 3 of 20 
studies, however, showed a significant difference in FFM loss between groups and only 1 of them included adults aged $>50$ years. Furthermore, the studies also included adults aged $<50$ years. Of major importance for the evaluation of FFM loss is weight loss over time [29-31]. Because energy deficit showed a positive association with FFM loss, the results also support that energy deficit is an important predictor and therefore a confounder in studies analyzing the effect of protein on weight loss. Improved satiety and an increased thermic effect of food on an HP diet $[32,33]$ facilitate weight loss on an HP diet and might therefore contribute to a higher energy deficit which prevents/or acts against the FFM-preserving effects of an HP diet. Weight loss was, however, not significantly higher in the HP diet group $(p=0.54)$.

Our assumption that the REE is better maintained following an increased protein intake during weight loss could not be confirmed. This is due to the lack of an effect of the HP diet on preservation of FFM. In addition, weight reduction may lead to an adaptive thermogenesis, indicating that the REE decline is independent of the loss of FFM [29, 34-36]. Here, there was a difference after adjustment of REE for FFM, and therefore an adaptive thermogenesis was present and the risk of weight regain was higher. Tang et al. [10] showed a significant better preservation of FFM after 12 weeks of weight loss in the HP group ( 1.4 vs. $0.8 \mathrm{~g} / \mathrm{kg}$ ), and macronutrients did not affect the reduction in REE in 43 overweight middle aged men. Luscombe et al. [37] also did not find an effect of diet composition ( 27 vs. $16 \%$ protein, 12-week intervention) on REE in adults (age 35-65 years). By contrast, Baba et al. [38] found a significant lower decrease in REE in the HP group (45 vs. $12 \%)$ in 13 men $(-132 \pm 51$ vs. $-384 \pm$ $85 \mathrm{kcal})$ after a 4 -week energy-restricted diet. Data of FFM changes, however, were not available (Table 5).

Similar to the present study, improvements in PF (SPPB score: $+2.4 \pm 1.7[\mathrm{HP}]$ vs. $+0.9 \pm 1.7[\mathrm{NP}] ; p<0.01$ ), but with group effects in favor of the HP group (1.2 vs. 0.8 $\mathrm{g} / \mathrm{kg}$ ) in adults aged $>60$ years, were shown after 6 months of weight reduction by $8 \mathrm{~kg}$ [7]. Verreijen et al. [13] also observed a significant improvement in walking speed and hand grip strength in 88 older subjects (age $>55$ years) after a weight loss of $3.5 \mathrm{~kg}$ within 13 weeks, without an impact of protein intake (1.11 and $0.85 \mathrm{~g} / \mathrm{kg}$ ). An HP diet $(1.3 \mathrm{~g} / \mathrm{kg})$ in combination with physical training led to greater improvements of these parameters within 10 weeks of weight reduction compared to an HP diet or training alone in older adults (age $>55$ years) [16]. In contrast, other studies found no improvement in walking speed in a weight loss group with $1.2 \mathrm{~g} / \mathrm{kg}$ protein versus a stable-weight group with $0.8 \mathrm{~g} / \mathrm{kg}$ in older adults (age > 65 years, 6-month intervention) [39]. Also muscle strength or PF changes remained unaffected after 12 weeks of energy restriction $(-25 \%)$ by protein intake $(1.7$ vs. $1.0 \mathrm{~g} / \mathrm{kg}$ ) in 61 adults aged $\geq 55$ years [15] (Table 6 ).

\section{Strengths and Limitations}

The present study protocol was based on the recommendations of Bellg et al. [40] for enhancement of treatment fidelity. We recorded a dropout rate of $15 \%$ during the intervention phase, which is quite similar to the rate of $15-20 \%$ in other studies $[13,39,41]$ and below $20 \%$, a value above which Schulz and Grimes [42] postulate a limited validity of study results. However, during followup the loss of subjects was $46 \%$. Therefore, these results should be interpreted with caution and further studies with a long-term follow-up period are needed. Nevertheless, this study was characterized by good compliance, demonstrated by the protein-induced increase in serum urea levels in the HP compared to the NP group as well as the low difference between the measured and the predicted weight loss. Dietary records indicated that the target protein intakes of 1.5 and $0.8 \mathrm{~g} / \mathrm{kg}$ were almost achieved, with $1.4 \pm 0.1$ and $0.8 \pm 0.1 \mathrm{~g} / \mathrm{kg}$ of protein per day in both groups. The differences in the recorded energy intake illustrate the problem of under- or overreporting, as there was no difference in weight loss. This double-blind RCT is characterized by an intensive assessment of dietary history and individual nutritional plans. The a priori measurement of REE by indirect calorimetry and the assessment of PAL allowed estimation of individual energy requirements and a personalized prescription for energy intake.

In conclusion, this study indicates that an energy-reduced HP diet in combination with 2 meal replacements and high-quality whey protein without physical exercise was more effective for maintaining PF measured by hand grip strength compared to an isocaloric weight loss diet with a normal protein intake in overweight, postmenopausal women. The energy deficit and the speed of weight loss have an important influence on preservation of FFM and should be more considered as a confounder in further studies. Physical exercise should be integrated into weight loss interventions whenever feasible for the subjects.

\section{Acknowledgement}

We thank all of the women for their participation. We would also like to thank the students and dieticians for their active support in conduction of this study. 


\section{Statement of Ethics}

This study complies with the guidelines for human studies and was conducted in accordance with the World Medical Association Declaration of Helsinki. The Ethics Committee of the Fulda University of Applied Sciences endorsed the study protocol, and written informed consent was obtained from all of the subjects. This study is registered in the German Clinical Trial Register (DRKS00011238).

\section{Conflict of Interest Statement}

The authors have no conflict of interests to declare.

\section{Funding Sources}

We acknowledge support from the Open Access Publishing Fund of the Fulda University of Applied Sciences. The shake powder was sponsored by the company Precon, and the whey protein powder was sponsored by the company Primal State. The preparation of data and this paper was carried out independently of the sponsors.

\section{Author Contributions}

I.E. created the study design, conducted this study, performed the statistical analysis, and wrote this paper. K.K.-M. supervised this study. A.B.-W., S.C.B., and K.K.-M. critically revised this paper. All of the authors read and approved the final version of this work.

\section{References}

1 Mensink GB, Schienkiewitz A, Haftenberger $\mathrm{M}$, Lampert $\mathrm{T}$, Ziese $\mathrm{T}$, Scheidt-Nave C. [Overweight and obesity in Germany: results of the German Health Interview and Examination Survey for Adults (DEGS1)]. Bundesgesundheitsblatt Gesundheitsforschung Gesundheitsschutz. 2013 May;56(5-6): 786-94.

2 Jensen GL, Hsiao PY. Obesity in older adults: relationship to functional limitation. Curr Opin Clin Nutr Metab Care. 2010 Jan;13(1): 46-51.

3 Prado CM, Wells JC, Smith SR, Stephan BC, Siervo M. Sarcopenic obesity: A Critical appraisal of the current evidence. Clin Nutr. 2012 Oct;31(5):583-601.

4 Villareal DT, Chode S, Parimi N, Sinacore DR, Hilton T, Armamento-Villareal R, et al. Weight loss, exercise, or both and physical function in obese older adults. N Engl J Med. 2011 Mar;364(13):1218-29.

5 Yadigar S, Yavuzer H, Yavuzer S, Cengiz M, Yürüyen M, Döventaş A, et al. Primary Sarcopenia in Older People with Normal Nutrition. J Nutr Health Aging. 2016 Mar;20(3):234-8.

6 Drey M, Maetzler W, Ferrari U. Sarkopenie. In: Maetzler W, Dodel R, Jacobs AH, editors. Neurogeriatrie: ICF-basierte Diagnose und Behandlung. Berlin, Heidelberg: Springer Berlin Heidelberg; 2019. pp. 69-84.

7 Porter Starr KN, Pieper CF, Orenduff MC, McDonald SR, McClure LB, Zhou R, et al. Improved Function With Enhanced Protein Intake per Meal: A Pilot Study of Weight Reduction in Frail, Obese Older Adults. J Gerontol A Biol Sci Med Sci. 2016 Oct;71(10):1369-75.

8 Bales CW, Porter Starr KN, Orenduff MC, McDonald SR, Molnar K, Jarman AK, et al. Influence of Protein Intake, Race, and Age on Responses to a Weight-Reduction Intervention in Obese Women. Curr Dev Nutr. 2017 May;1(5):e000703.
9 Leidy HJ, Carnell NS, Mattes RD, Campbell WW. Higher protein intake preserves lean mass and satiety with weight loss in pre-obese and obese women. Obesity (Silver Spring). 2007 Feb;15(2):421-9.

10 Tang M, Armstrong CL, Leidy HJ, Campbell WW. Normal vs. high-protein weight loss diets in men: effects on body composition and indices of metabolic syndrome. Obesity (Silver Spring). 2013 Mar;21(3):E204-10.

11 Farnsworth E, Luscombe ND, Noakes M, Wittert G, Argyiou E, Clifton PM. Effect of a high-protein, energy-restricted diet on body composition, glycemic control, and lipid concentrations in overweight and obese hyperinsulinemic men and women. Am J Clin Nutr. 2003 Jul;78(1):31-9.

12 Gordon MM, Bopp MJ, Easter L, Miller GD, Lyles MF, Houston DK, et al. Effects of dietary protein on the composition of weight loss in post-menopausal women. J Nutr Health Aging. 2008 Oct;12(8):505-9.

13 Verreijen AM, Verlaan S, Engberink MF, Swinkels S, de Vogel-van den Bosch J, Weijs PJ. A high whey protein-, leucine-, and vitamin D-enriched supplement preserves muscle mass during intentional weight loss in obese older adults: a double-blind randomized controlled trial. Am J Clin Nutr. 2015 Feb;101(2):279-86.

14 Beavers KM, Gordon MM, Easter L, Beavers DP, Hairston KG, Nicklas BJ, et al. Effect of protein source during weight loss on body composition, cardiometabolic risk and physical performance in abdominally obese, older adults: a pilot feeding study. J Nutr Health Aging. $2015 \mathrm{Jan} ; 19(1): 87-95$.

15 Backx EM, Tieland M, Borgonjen-van den Berg KJ, Claessen PR, van Loon LJ, de Groot LC. Protein intake and lean body mass preservation during energy intake restriction in overweight older adults. Int J Obes. 2016 Feb; 40(2):299-304.
16 Verreijen AM, Engberink MF, Memelink RG, van der Plas SE, Visser M, Weijs PJ. Effect of a high protein diet and/or resistance exercise on the preservation of fat free mass during weight loss in overweight and obese older adults: a randomized controlled trial. Nutr J. 2017 Feb;16(1):10.

17 Schollenberger AE, Karschin J, Meile T, Küper MA, Königsrainer A, Bischoff SC. Impact of protein supplementation after bariatric surgery: A randomized controlled doubleblind pilot study. Nutrition. 2016 Feb;32(2): 186-92.

18 Bosy-Westphal A, Wolf A, Bührens F, Hitze B, Czech N, Mönig H, et al. Familial influences and obesity-associated metabolic risk factors contribute to the variation in resting energy expenditure: the Kiel Obesity Prevention Study. Am J Clin Nutr. 2008 Jun;87(6):1695-701.

19 Bosy-Westphal A, Schautz B, Lagerpusch M, Pourhassan M, Braun W, Goele K, et al. Effect of weight loss and regain on adipose tissue distribution, composition of lean mass and resting energy expenditure in young overweight and obese adults. Int J Obes. 2013 Oct; 37(10):1371-7.

20 Deutsche Gesellschaft für Ernährung. Referenzwerte für die Nährstoffzufuhr. 2. Auflage, 2., aktualisierte Ausgabe. Frankfurt am Main: Umschau; 2015.

21 Guralnik JM, Simonsick EM, Ferrucci L, Glynn RJ, Berkman LF, Blazer DG, et al. A short physical performance battery assessing lower extremity function: association with self-reported disability and prediction of mortality and nursing home admission. J Gerontol. 1994 Mar;49(2):M85-94.

22 Roberts HC, Denison HJ, Martin HJ, Patel $\mathrm{HP}$, Syddall H, Cooper C, et al. A review of the measurement of grip strength in clinical and epidemiological studies: towards a standardised approach. Age Ageing. $2011 \mathrm{Jul}$; 40(4):423-9. 
23 Hall KD, Sacks G, Chandramohan D, Chow CC, Wang YC, Gortmaker SL, et al. Quantification of the effect of energy imbalance on bodyweight. Lancet. 2011 Aug;378(9793): 826-37.

24 Goele K, Bosy-Westphal A, Kossel E, Glüer C, Heller M, Rümcker B, et al. Relative Validität und Präzision der Bioelektrischen Impedanzanalyse zur Erfassung von Veränderungen in der Körperzusammensetzung bei adipösen Patientinnen vor und nach einer Gewichtsreduktion. Akt Ernähr Med. 2008;33(06): 284-90.

25 Layman DK, Evans EM, Erickson D, Seyler J, Weber J, Bagshaw D, et al. A moderate-protein diet produces sustained weight loss and long-term changes in body composition and blood lipids in obese adults. J Nutr. 2009 Mar; 139(3):514-21.

26 Flechtner-Mors M, Boehm BO, Wittmann R, Thoma U, Ditschuneit HH. Enhanced weight loss with protein-enriched meal replacements in subjects with the metabolic syndrome. Diabetes Metab Res Rev. 2010 Jul; 26(5):393-405.

27 Johnston CS, Sears B, Perry M, Knurick JR. Use of Novel High-Protein Functional Food Products as Part of a Calorie-Restricted Diet to Reduce Insulin Resistance and Increase Lean Body Mass in Adults: A Randomized Controlled Trial. Nutrients. 2017 Oct; 9(11):E1182.

28 Kim JE, O'Connor LE, Sands LP, Slebodnik $\mathrm{MB}$, Campbell WW. Effects of dietary protein intake on body composition changes after weight loss in older adults: a systematic review and meta-analysis. Nutr Rev. 2016 Mar;74(3): $210-24$.

29 Müller MJ, Enderle J, Braun W, Pourhassan M, Bosy-Westphal A. Gibt es die Adaptive Thermogenese? Adipositas - Ursachen, Folgeerkrankungen. Therapie. 2015;09(04): 210-6.
30 Garthe I, Raastad T, Refsnes PE, Koivisto A, Sundgot-Borgen J. Effect of two different weight-loss rates on body composition and strength and power-related performance in elite athletes. Int J Sport Nutr Exerc Metab. 2011 Apr;21(2):97-104.

31 Ashtary-Larky D, Ghanavati M, LamuchiDeli N, Payami SA, Alavi-Rad S, Boustaninejad M, et al. Rapid Weight Loss vs. Slow Weight Loss: Which is More Effective on Body Composition and Metabolic Risk Factors? Int J Endocrinol Metab. 2017 May; 15(3):e13249.

32 Bendtsen LQ, Lorenzen JK, Bendsen NT, Rasmussen C, Astrup A. Effect of dairy proteins on appetite, energy expenditure, body weight, and composition: a review of the evidence from controlled clinical trials. Adv Nutr. 2013 Jul;4(4):418-38.

33 Leidy HJ, Tang M, Armstrong CL, Martin CB, Campbell WW. The effects of consuming frequent, higher protein meals on appetite and satiety during weight loss in overweight/obese men. Obesity (Silver Spring). 2011 Apr;19(4): 818-24.

34 Goele K, Bosy-Westphal A, Rumcker B, Lagerpusch M, Muller MJ. Influence of changes in body composition and adaptive thermogenesis on the difference between measured and predicted weight loss in obese women. Obes Facts. 2009;2(2):105-9.

35 Leibel RL, Rosenbaum M, Hirsch J. Changes in energy expenditure resulting from altered body weight. N Engl J Med. 1995 Mar;332(10): 621-8.

36 Fothergill E, Guo J, Howard L, Kerns JC, Knuth ND, Brychta R, et al. Persistent metabolic adaptation 6 years after "The Biggest Loser" competition. Obesity (Silver Spring). 2016 Aug;24(8):1612-9.

37 Luscombe ND, Clifton PM, Noakes M, Farnsworth E, Wittert G. Effect of a high-protein, energy-restricted diet on weight loss and en- ergy expenditure after weight stabilization in hyperinsulinemic subjects. Int J Obes Relat Metab Disord. 2003 May;27(5):582-90.

38 Baba NH, Sawaya S, Torbay N, Habbal Z Azar S, Hashim SA. High protein vs high carbohydrate hypoenergetic diet for the treatment of obese hyperinsulinemic subjects. Int J Obes Relat Metab Disord. 1999 Nov;23(11): 1202-6.

39 Beavers KM, Nesbit BA, Kiel JR, Sheedy JL, Arterburn LM, Collins AE, et al. Effect of an Energy-Restricted, Nutritionally Complete, Higher Protein Meal Plan on Body Composition and Mobility in Older Adults With Obesity: A Randomized Controlled Trial. J Gerontol A Biol Sci Med Sci. 2019 May;74(6): 929-35.

40 Bellg AJ, Borrelli B, Resnick B, Hecht J, Minicucci DS, Ory M, et al.; Treatment Fidelity Workgroup of the NIH Behavior Change Consortium. Enhancing treatment fidelity in health behavior change studies: best practices and recommendations from the NIH Behavior Change Consortium. Health Psychol. 2004 Sep;23(5):443-51.

41 Wright CS, Zhou J, Sayer RD, Kim JE, Campbell WW. Effects of a High-Protein Diet Including Whole Eggs on Muscle Composition and Indices of Cardiometabolic Health and Systemic Inflammation in Older Adults with Overweight or Obesity: A Randomized Controlled Trial. Nutrients. 2018 Jul;10(7):E946.

42 Schulz KF, Grimes DA. Sample size slippages in randomised trials: exclusions and the lost and wayward. Lancet. 2002 Mar;359(9308): 781-5.

43 Wycherley TP, Buckley JD, Noakes M, Clifton PM, Brinkworth GD. Comparison of the effects of weight loss from a high-protein versus standard-protein energy-restricted diet on strength and aerobic capacity in overweight and obese men. Eur J Nutr. 2013 Feb;52(1): $317-25$. 